\title{
Charge-density-wave origin of cuprate checkerboard visualized by scanning tunnelling microscopy
}

\author{
W. D. WISE ${ }^{1}$, M. C. BOYER ${ }^{1}$, KAMALESH CHATTERJEE ${ }^{1}$, TAKESHI KOND01,2* T. TAKEUCH ${ }^{2,3}$, H. IKUTA², \\ YAYU WANG ${ }^{1 *}$ AND E. W. HUDSON ${ }^{1 \dagger}$
}

${ }^{1}$ Department of Physics, Massachusetts Institute of Technology, Cambridge, Massachusetts 02139, USA

${ }^{2}$ Department of Crystalline Materials Science, Nagoya University, Nagoya 464-8603, Japan

${ }^{3}$ EcoTopia Science Institute, Nagoya University, Nagoya 464-8603, Japan

*Present address: Ames Laboratory and Department of Physics and Astronomy, lowa State University, Ames, lowa 50011, USA (T.K.); Department of Physics, Tsinghua University, Beijing 100084, China (Y.W.)

†e-mail: ehudson@mit.edu

One of the main challenges in understanding high- $T_{c}$ superconductivity is to disentangle the rich variety of states of matter that may coexist, cooperate or compete with $d$-wave superconductivity. At centre stage is the pseudogap phase, which occupies a large portion of the cuprate phase diagram surrounding the superconducting dome ${ }^{1}$. Using scanning tunnelling microscopy, we find that a static, non-dispersive, 'checkerboard'-like electronic modulation exists in a broad regime of the cuprate phase diagram and exhibits strong doping dependence. The continuous increase of checkerboard periodicity with hole density strongly suggests that the checkerboard originates from charge-density-wave formation in the antinodal region of the cuprate Fermi surface. These results reveal a coherent picture for static electronic orderings in the cuprates and shed important new light on the nature of the pseudogap phase.

A great deal of current interest is focused on the 'checkerboard'like electronic lattices first discovered in cuprates by scanning tunnelling microscopy (STM) in vortex cores in optimally doped $\mathrm{Bi}_{2} \mathrm{Sr}_{2} \mathrm{CaCu}_{2} \mathrm{O}_{8+\delta}$ (Bi-2212) (ref. 2). This ordering was found to have a roughly 4 unit-cell $\left(4 a_{0}\right)$ wavelength orientated along the $\mathrm{Cu}-\mathrm{O}$ bond direction. Subsequent STM investigations of the cuprates have revealed other checkerboard structures in the absence of a magnetic field. For example, in the superconducting state of Bi-2212, the first report of a checkerboard saw a roughly $4 a_{0}$ wavelength throughout the sample ${ }^{3}$, whereas a later study found the ordering (wavelength $4.5 a_{0}$ ) limited to regions with large-gap ('zero-temperature pseudogap') tunnelling spectra ${ }^{4}$. A checkerboard was also found in slightly underdoped Bi-2212 above the superconducting transition temperature $T_{c}$ with wavelength $4.7 a_{0} \pm 0.2 a_{0}$ (ref. 5). In $\mathrm{Ca}_{2-x} \mathrm{Na}_{x} \mathrm{CuO}_{2} \mathrm{Cl}_{2}(\mathrm{Na}$ CCOC), a commensurate electronic crystal phase with period $4 a_{0}$ was found at low temperatures in both superconducting and nonsuperconducting samples ${ }^{6}$.

Although it is not yet clear whether these checkerboards all represent the same electronic entities, many models have been proposed to explain the mechanisms of these novel electronic phases and their implications for the pseudogap and high- $T_{\mathrm{c}}$ superconductivity $^{7-17}$. Initially, it was suggested ${ }^{2,9}$ that the $4 a_{0}$ pattern in Bi-2212 vortex cores is the charge-density modulation accompanying the $8 a_{0}$ spin-density wave (SDW) created by an external magnetic field. Other explanations of checkerboards include exotic orderings such as fluctuating one-dimensional stripes $^{12}$, modulations of electron hopping amplitude ${ }^{13}$, Cooper pair Wigner crystal $^{14}$ or density wave ${ }^{15}$ and orbital-currentinduced $d$-density wave ${ }^{17}$. Recently, angle-resolved photoemission spectroscopy (ARPES) on Na-CCOC found parallel Fermi surface segments with a nesting vector around $2 \pi / 4 a_{0}$ in the antinodal region, suggesting charge-density-wave (CDW) formation as the origin of the checkerboard ${ }^{18}$. Unfortunately, existing data are inadequate to discriminate between the different models, mainly because the experiments were carried out on small, isolated regions of the complex cuprate phase diagram.

Here, we report on systematic doping- and temperaturedependent STM studies of charge-density modulations in the high-temperature superconductor $\mathrm{Bi}_{2-y} \mathrm{~Pb}_{\mathrm{y}} \mathrm{Sr}_{2-z} \mathrm{La}_{\mathrm{z}} \mathrm{CuO}_{6+x}$ $(\mathrm{Pb}-$ and La-substituted $\mathrm{Bi}-2201)$ (ref. 19). We find that a static (non-fluctuating), non-dispersive (energy-independent), checkerboard-like electronic lattice exists over a wide range of doping, and that its wavelength increases with increasing hole density. This unexpected trend strongly supports the physical picture of Fermi-surface-nesting-induced CDW formation and is corroborated by comparison to band-structure calculations and ARPES measurements.

These experiments were conducted on a home-built variable-temperature STM, which enables simultaneous mapping of atomic-scale topography and differential conductance spectroscopy, proportional to the energy-dependent local density of states (LDOS) of the sample. We begin by describing our results on optimally doped Bi-2201 with $T_{\mathrm{c}}=35 \mathrm{~K}$. Figure 1a shows a typical atomic-resolution STM topography of a 785- $\AA$ region measured at $T=6 \mathrm{~K}$. The inset shows the $\mathrm{Pb}$ (brighter) and $\mathrm{Bi}$ (dimmer) atoms of the exposed $\mathrm{BiO}$ plane. The $\mathrm{CuO}_{2}$ plane lies $\sim 5 \AA$ below. A representative differential conductance spectrum from this area 

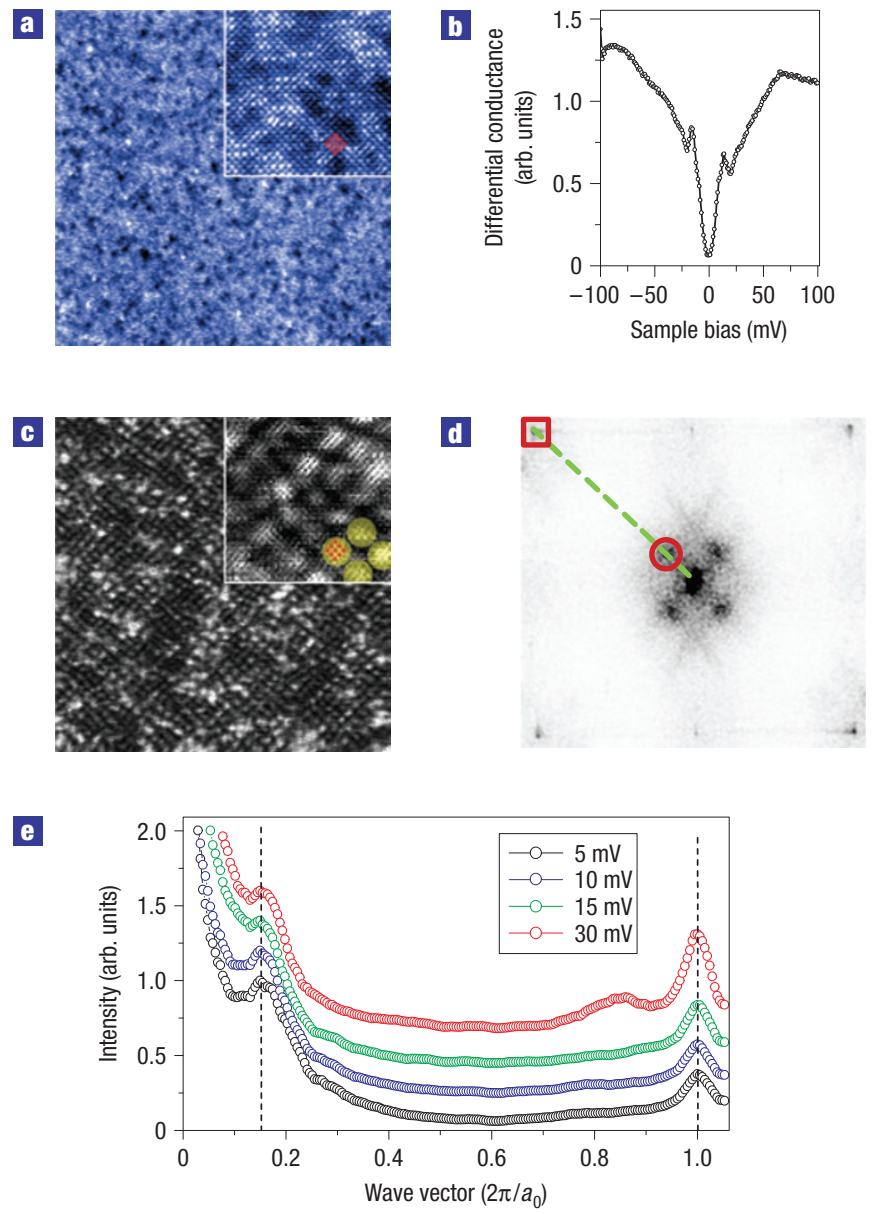

Figure 1 Anatomy of the checkerboard in optimally doped Bi-2201. a, STM topography of a $785-\AA$ region of optimally doped $\left(T_{\mathrm{c}}=35 \mathrm{~K}\right) \mathrm{Bi}-2201$ measured at $T=6 \mathrm{~K}$. The magnified inset $(110 \AA)$ (and red dots on 9 atoms) show the clear atomic lattice in this high-resolution data. $\mathbf{b}$, Spatially averaged differential conductance spectra measured in the area shown in a exhibit two distinct gaps: a superconducting gap $\Delta_{\mathrm{SC}} \sim 15 \mathrm{meV}$ and pseudogap $\Delta_{\mathrm{PG}} \sim 75 \mathrm{meV}$.

c, Conductance maps, here taken with bias voltage $10 \mathrm{meV}$ on the same region as a, show a checkerboard structure in the LDOS with a wavelength much larger than the atomic lattice. The inset is magnified as in a, with the same 9 atoms highlighted in red. Four checkerboard maxima are also highlighted (yellow) for clarity. d, Fourier transform of the map shown in c. The checkerboard wave vectors (circled) appear as four spots along the same direction as the atomic lattice (outlined with a square). The dashed line shows the locations of the line cuts in e. e, Line cuts extracted from Fourier-transform LDOS maps with different bias voltages. The left vertical line marks the position of the checkerboard wave vector, $2 \pi / 6.2 a_{0}$ for all energies, and the right vertical line indicates the atomic lattice wave vector $2 \pi / a_{0}$. All data in this letter was acquired with feedback setpoint parameters $I_{S}=400 \mathrm{pA}$ and $V_{\mathrm{S}}=-100 \mathrm{mV}$ or $V_{\mathrm{S}}=-200 \mathrm{mV}$.

(Fig. 1b) has a clear inner gap with peaks near $15 \mathrm{meV}$, probably associated with the superconducting gap, and a pseudogap with size roughly $75 \mathrm{meV}$ (ref. 20). A differential conductance map of the region taken at a bias of $10 \mathrm{meV}$ (Fig. 1c) shows a checkerboard-like electronic lattice, strikingly similar to those observed in other cuprates $^{3-6}$. The checkerboard is observed to beyond $50 \mathrm{meV}$ at both positive and negative sample bias, although the pattern appears most strongly at low, positive bias. It appears in maps taken with feedback setpoint voltages ranging from 10 to $300 \mathrm{mV}$,
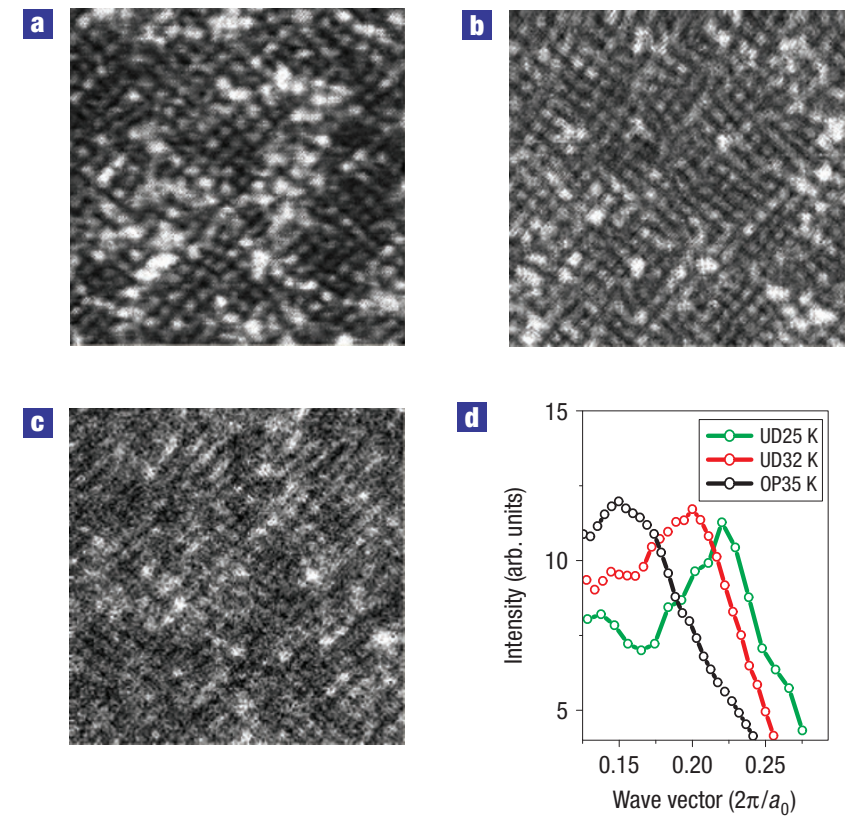

Figure 2 Doping dependence of the checkerboard. a-c, $400 \AA$ conductance maps of optimally doped, $T_{\mathrm{c}}=35 \mathrm{~K}(\mathbf{a})$, underdoped, $T_{\mathrm{c}}=32 \mathrm{~K}(\mathbf{b})$, and underdoped, $T_{\mathrm{c}}=25 \mathrm{~K}(\mathrm{c}), \mathrm{Bi}-2201$. All maps were taken with $10 \mathrm{mV}$ sample bias at $T=6 \mathrm{~K}$. The checkerboard structures shown in $\mathbf{b}, \mathbf{c}$ have denser packing than in $\mathbf{a}$, indicating a shorter wavelength in underdoped samples. $\mathbf{d}$, Line cuts along the atomic lattice direction of the Fourier-transform LDOS maps of the three samples. The cuts peak at the checkerboard wave vectors, corresponding to wavelengths of $6.2 a_{0}, 5.1 a_{0}$ and $4.5 a_{0}$, respectively.

with feedback currents from 50 to $800 \mathrm{pA}$, and in topographic scans at $10 \mathrm{mV}$ bias.

The wavelength of this checkerboard is determined from the Fourier transform of the image, as shown in Fig. 1d, where the checkerboard appears as four peaks (one is circled). Its wave vector corresponds to a wavelength $d \sim 6.2 a_{0} \pm 0.2 a_{0}$, much larger than that of any such structure previously reported. Figure $1 \mathrm{e}$ shows a line cut along the atomic lattice $(\pi, 0)$ direction of the Fourier-transform LDOS maps taken at different bias voltages. The consistent position of the checkerboard wave vector observed at different energies, marked by the left dashed line, indicates that the checkerboard is a non-dispersive, static ordering.

We find similar checkerboard structures in underdoped Bi-2201 samples with $T_{\mathrm{c}}=32 \mathrm{~K}$ (Fig. 2b) and $T_{\mathrm{c}}=25 \mathrm{~K}$ (Fig. 2c). Surprisingly, Fourier transforms reveal that checkerboard periodicities in these underdoped samples are reduced to $5.1 a_{0} \pm 0.2 a_{0}$ and $4.5 a_{0} \pm 0.2 a_{0}$ respectively, significantly shorter than in the optimally doped sample. This can be seen directly from the denser packing of the underdoped checkerboard (Fig. 2b,c) compared with that in the optimally doped one (Fig. 2a). Figure $2 \mathrm{~d}$ summarizes this doping dependence in line cuts of the Fourier transforms along the atomic lattice $(\pi, 0)$ direction. The increase of the checkerboard wave vector with decreasing hole density is pronounced.

In contrast to doping, temperature has no measurable effect on the checkerboard wave vector. The LDOS map of the underdoped $T_{\mathrm{c}}=32 \mathrm{~K}$ sample measured at $35 \mathrm{~K}$ (Fig. $3 \mathrm{a}$ ) is qualitatively the same as that measured at $6 \mathrm{~K}$ (Fig. 2a). Figure $3 \mathrm{~b}$ shows line cuts of the Fourier transforms of maps measured at a wide range of temperatures, demonstrating that the peak location is unaffected by temperature and in particular $T_{c}$. 

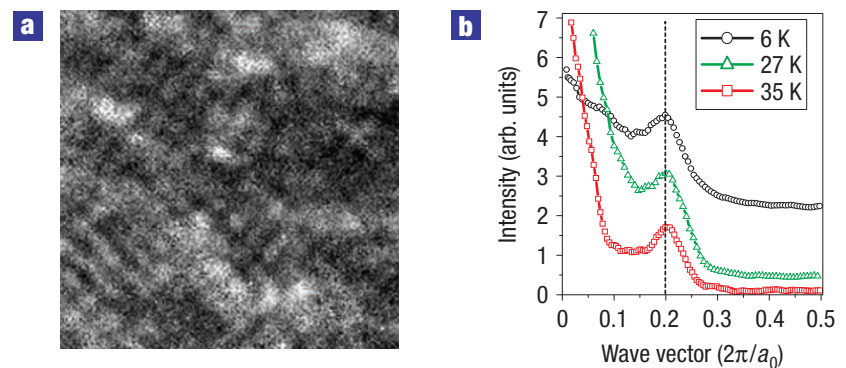

Figure 3 Temperature independence of the checkerboard. a, $300 \AA, 10 \mathrm{mV}$ conductance map of the underdoped $T_{\mathrm{c}}=32 \mathrm{~K}$ sample measured at $T=35 \mathrm{~K}$, slightly above $T_{\mathrm{c}}$. The checkerboard is qualitatively unchanged from low temperatures (Fig. 2b). b, Line cuts along the atomic lattice direction of Fourier-transform LDOS maps at this and lower temperatures indicate that the checkerboard wave vector is temperature independent (vertical line).

These results reveal important new features of the checkerboard. First, the non-dispersive charge-density modulation found previously in Bi-2212 (refs 3-5) and $\mathrm{Na}-\mathrm{CCOC}$ (ref. 6) also exists in Bi-2201, suggesting that it is a robust feature that prevails in the cuprate phase diagram, in both optimally doped and underdoped phases, and at temperatures both below and above $T_{\mathrm{c}}$. More importantly, the doping dependence of the checkerboard periodicity puts stringent constraints on relevant theoretical models, as discussed below.

We first emphasize that the checkerboard structures reported here and previously $y^{3-6}$ are distinct from the spatial LDOS modulations induced by quasiparticle interference ${ }^{21}(\mathrm{QPI})$. QPI wave vectors depend strongly on energy because they are formed by interference of elastically scattered quasiparticles residing on equal-energy contours of the Fermi surface ${ }^{22}$. In contrast, the checkerboard lattice is non-dispersive. Quantitatively, modelling QPI (ref. 22) using a tight-binding Fermi surface ${ }^{23}$ and $\Delta=\Delta_{0} \cos (2 \theta)$ with $\Delta_{0}=15 \mathrm{mV}$ suggests that the QPI vector closest to these checkerboard peaks would disperse from about 0.18 $\left(2 \pi / a_{0}\right)$ to $0.33\left(2 \pi / a_{0}\right)$ over the energy range shown in Fig. 1 , clearly inconsistent with the non-dispersing vectors we report here. Similarly, QPI from the ends of the Fermi $\operatorname{arcs}^{24}$, a seemingly reasonable explanation of the checkerboard observed strictly above $T_{\mathrm{c}}$ in Bi-2212 (ref. 5), cannot explain the temperature-independent, non-dispersive pattern reported here. Although we have observed wave vectors associated with QPI Bi-2201, we defer discussion of these interesting features to a future paper.

For non-dispersive checkerboard formation, a number of explanations have been proposed. Most predict a checkerboard wavelength that decreases with doping, opposite to our results. For example, the presence of a $4 a_{0}$ checkerboard in $\mathrm{Bi}-2212$ vortex cores $^{2}$ was initially attributed ${ }^{2,7-11}$ to the long-sought concomitant charge modulation of the $8 a_{0}$ SDW found earlier by neutron scattering in the vortex lattice state of $\mathrm{La}_{2-x} \mathrm{Sr}_{x} \mathrm{CuO}_{4}$ (ref. 25). In a variety of cuprates, neutron scattering experiments have found incommensurate SDWs, which should create accompanying charge-density modulations with half the wavelength ${ }^{7-11}$. Although this is an appealing picture for the vortex checkerboard, that the measured SDW wavelength decreases with increasing doping ${ }^{26}$ means it cannot explain the patterns reported here.

The stripe model $^{12}$, which posits that fluctuating stripes can form a checkerboard-like pattern when pinned by impurities, also predicts that wavelengths should decrease with doping, as more holes means more stripes and a smaller average distance between

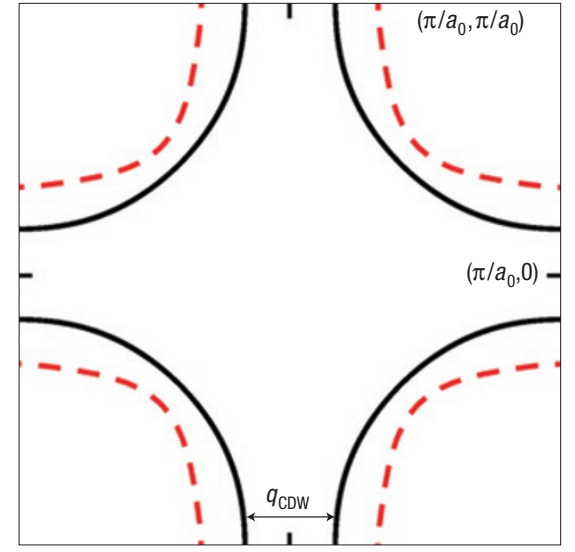

Figure 4 Fermi surface nesting. Tight-binding-calculated Fermi surface (solid black curve) of optimally doped Bi-2201 (ref. 23) based on ARPES data ${ }^{33}$. The nesting wave vector (black arrow) in the antinodal flat band region has length $2 \pi / 6.2 a_{0}$. Underdoped Bi-2201 Fermi surfaces (shown schematically as red dashed lines) show a reduced volume and longer nesting wave vector, consistent with a CDW origin of the doping-dependent checkerboard pattern reported here.

them. Similarly, in theories that attribute checkerboard patterns to real-space organization of Coulomb-repulsed Cooper pairs in the form of Wigner crystals ${ }^{14}$ or Cooper pair density waves ${ }^{15}$, the distance between neighbouring Cooper pairs would be expected to decrease with doping as the Cooper pairs become less dilute. These predictions are also at odds with our observations.

We propose that the most likely origin for the checkerboard is the formation of an incommensurate CDW (ref. 16). The cuprate Fermi surface flattens out in the antinodal $(0, \pi)$ region and forms parallel ('nested') sections there (Fig. 4). Nesting benefits the formation of CDWs, as a modulation at a single wave vector can gap large sections of the Fermi surface and lower electronic energy. With fewer holes in the $\mathrm{CuO}_{2}$ plane in underdoped samples, the cuprate hole-like Fermi surface shrinks (dashed line), leading to a larger nesting wave vector and hence smaller real-space wavelength, in agreement with the doping dependence we report here.

The CDW picture can also explain the checkerboards in Bi-2212 and Na-CCOC. ARPES measurements on slightly underdoped Bi-2212 reveal an $\sim 2 \pi / 5 a_{0}$ antinodal nesting wave vector $^{27}$, in agreement with the STM-measured $4.7 a_{0}$ checkerboard periodicity ${ }^{5}$. In Na-CCOC, the match of the $4 a_{0}$ checkerboard wavelength to their ARPES-measured nesting wave vector around $2 \pi / 4 a_{0}$ also led researchers to the conclusion of a CDW (ref. 18). The doping dependence sought in that study, but absent in the invariably commensurate $4 a_{0}$ checkerboard, possibly owing to lock-in of an incommensurate CDW by the crystal lattice, is revealed here in $\mathrm{Bi}-2201$, where the checkerboard is incommensurate and strongly doping dependent, clearly favouring a Fermi-surface-nesting-induced CDW picture.

With a CDW as the most likely source of the checkerboard, we next turn our attention to the relationship between this CDW and other physics in the system. In particular, because we observe it both above and below $T_{c}$, the question arises as to whether the CDW is the hidden order of the pseudogap phase. Recent discovery of a dichotomy between the nodal and antinodal quasiparticles, revealed by Raman and ARPES experiment ${ }^{28-30}$, supports this conjecture. Quasiparticles near the node have a $d$-wave gap that opens at $T_{\mathfrak{c}}$, and hence are assumed to be responsible for $d$-wave superconductivity. Quasiparticles near the antinode on the other 
hand have a large gap that is roughly temperature independent near, and exists well above, $T_{c}$. That this gap persists above $T_{c}$ demonstrates that it is the pseudogap. That this gap exists near the antinodes, where the nested Fermi surface seems responsible for the formation of the CDW we report here, suggests that the pseudogap may be the CDW gap.

Objecting to this claim, some have commented that a CDW gap need not be centred on the Fermi energy and thus at least at some dopings the pseudogap should be asymmetric around it. Close observation of our spectra affirms that the pseudogap is rarely symmetric about the Fermi energy. Figure $1 \mathrm{~b}$ shows peaks at $-88 \mathrm{meV}$ and $+66 \mathrm{meV}$, very asymmetric particularly considering the clear symmetry of the inner (superconducting gap) peaks at $\pm 15 \mathrm{meV}$. This asymmetry is ubiquitous in large-gap regions (see for example Fig. 1a of ref. 5 and Fig. 2b of ref. 4), where the pseudogap is clearly distinguishable from the symmetric superconducting gap, blunting this objection to the picture of the pseudogap as the CDW gap.

Although a nesting-driven CDW explanation of the checkerboard is generally consistent with our data, there remain unresolved issues that require further experimental and theoretical exploration. ARPES measurements of the Fermi surface nesting vector of $\mathrm{Bi}-2201$ by different groups contain significant disagreements, making quantitative numerical comparisons of the checkerboard wave vector to the nesting wave vector across dopings difficult (although the qualitative doping dependence is certainly in agreement with our results). ARPES also suggests that the antinodal states in the cuprates may be incoherent, and the mechanism by which a CDW would arise from incoherent states is unclear.

Furthermore, if a CDW existed in these samples, it could in principle be observed in scattering experiments, but no such observation has yet been reported. This may in part be due to the weak, glassy nature of these modulations ${ }^{31}$. Although X-ray has successfully detected charge modulations in $\mathrm{La}_{2-x} \mathrm{Ba}_{x} \mathrm{CuO}_{4}$, the inplane correlation length there $(\xi \sim 500 \AA$ ) (ref. 32$)$ is significantly longer than in Bi-2212 ( $\xi \sim 90 \AA)$ (ref. 5), Na-CCOC $(\xi \sim 40 \AA)$ (ref. 6) and in the Bi-2201 samples discussed here $(\xi \sim 35 \AA)$, perhaps owing to pinning by disorder in these samples. This difference alone would be responsible for a drop in scattering signal of a couple orders of magnitude for some techniques, and may thus explain the lack of corroborating results from scattering experiments.

Thus, despite similarities between CDWs, the checkerboard and features of the pseudogap, much work remains to be done before confirming or refuting this picture. The pseudogap is a rich phase, exhibiting a wide variety of phenomena and, so far, no theory has consistently explained all of the results of the large number of experimental probes of its nature. That the CDW discussed here explains some of them is a beginning.

Received 31 March 2008; accepted 6 June 2008; published 6 July 2008.

\section{References}

1. Timusk, T. \& Statt, B. The pseudogap in high-temperature superconductors: An experimental survey. Rep. Prog. Phys. 62, 61-122 (1999).

2. Hoffman, J. E. et al. A four unit cell periodic pattern of quasi-particle states surrounding vortex cores in $\mathrm{Bi}_{2} \mathrm{Sr}_{2} \mathrm{CaCu}_{2} \mathrm{O}_{8+\delta}$. Science 295, 466-469 (2002).
3. Howald, C., Eisaki, H., Kaneko, N., Greven, M. \& Kapitulnik, A. Periodic density-of-states modulations in superconducting $\mathrm{Bi}_{2} \mathrm{Sr}_{2} \mathrm{CaCu}_{2} \mathrm{O}_{8+\delta}$. Phys. Rev. B 67, 014533 (2003).

4. McElroy, K. et al. Coincidence of checkerboard charge order and antinodal state decoherence in strongly underdoped superconducting $\mathrm{Bi}_{2} \mathrm{Sr}_{2} \mathrm{CaCu}_{2} \mathrm{O}_{8+\delta}$. Phys. Rev. Lett. 94, 197005 (2005).

5. Vershinin, $\mathrm{M}$. et al. Local ordering in the pseudogap state of the high- $T_{\mathrm{c}}$ superconductor $\mathrm{Bi}_{2} \mathrm{Sr}_{2} \mathrm{CaCu}_{2} \mathrm{O}_{8+\delta}$. Science 303, 1995-1998 (2004).

6. Hanaguri, T. et al. A 'checkerboard' electronic crystal state in lightly hole-doped $\mathrm{Ca}_{2-x} \mathrm{Na}_{x} \mathrm{CuO}_{2} \mathrm{Cl}_{2}$. Nature 430, 1001-1005 (2004).

7. Chen, Y. \& Ting, C. S. Magnetic-field-induced spin-density wave in high-temperature superconductors. Phys. Rev. B 65, 180513 (2002).

8. Franz, M., Sheehy, D. E. \& Tesanovic, Z. Magnetic field induced charge and spin instabilities in cuprate superconductors. Phys. Rev. Lett. 88, 257005 (2002).

9. Sachdev, S. \& Zhang, S. C. Tuning order in cuprate superconductors. Science 295, 452-454 (2002).

10. Zhang, Y., Demler, E. \& Sachdev, S. Competing orders in a magnetic field: Spin and charge order in the cuprate superconductors. Phys. Rev. B 66, 094501 (2002).

11. Zhu, J. X., Martin, I. \& Bishop, A. R. Spin and charge order around vortices and impurities in high- $T_{c}$ superconductors. Phys. Rev. Lett. 89, 067003 (2002).

12. Kivelson, S. A. et al. How to detect fluctuating stripes in the high-temperature superconductors. Rev Mod. Phys. 75, 1201-1241 (2003).

13. Podolsky, D., Demler, E., Damle, K. \& Halperin, B. I. Translational symmetry breaking in the superconducting state of the cuprates: Analysis of the quasiparticle density of states. Phys. Rev. B 67, 094514 (2003).

14. Chen, H.-D., Vafek, O., Yazdani, A. \& Zhang, S.-C. Pair density wave in the pseudogap state of high temperature superconductors. Phys. Rev. Lett. 93, 187002 (2004).

15. Tesanovic, Z. Charge modulation, spin response, and dual Hofstadter butterfly in high- $T_{\mathrm{c}}$ cuprates. Phys. Rev. Lett. 93, 217004 (2004).

16. Li, J.-X., Wu, C.-Q. \& Lee, D.-H. Checkerboard charge density wave and pseudogap of high-T cuprate. Phys. Rev. B 74, 184515 (2006).

17. Seo, K., Chen, H.-D. \& Hu, J. d-wave checkerboard order in cuprates. Phys. Rev. B 76, 020511 (2007).

18. Shen, K. M. et al. Nodal quasiparticles and antinodal charge ordering in $\mathrm{Ca}_{2-x} \mathrm{Na}_{x} \mathrm{CuO}_{2} \mathrm{Cl}_{2}$. Science 307, 901-904 (2005).

19. Kondo, T. et al. Contribution of electronic structure to thermoelectric power in $(\mathrm{Bi}, \mathrm{Pb})_{2}(\mathrm{Sr}, \mathrm{La})_{2} \mathrm{CuO}_{6+\delta}$. Phys. Rev. B 72, 024533 (2005).

20. Boyer, M. C. et al. Imaging the two gaps of the high-temperature superconductor $\mathrm{Bi}_{2} \mathrm{Sr}_{2} \mathrm{CuO}_{6+x}$. Nature Phys. 3, 802-806 (2007).

21. Hoffman, J. E. et al. Imaging quasiparticle interference in $\mathrm{Bi}_{2} \mathrm{Sr}_{2} \mathrm{CaCu}_{2} \mathrm{O}_{8+\delta}$. Science 297, 1148-1151 (2002)

22. Wang, Q.-H. \& Lee, D.-H. Quasiparticle scattering interference in high-temperature superconductors. Phys. Rev. B 67, 020511 (2003).

23. Markiewicz, R. S., Sahrakorpi, S., Lindroos, M., Lin, H. \& Bansil, A. One-band tight-binding model parametrization of the high- $T_{\mathrm{c}}$ cuprates including the effect of $\mathrm{k}(\mathrm{z})$ dispersion. Phys. Rev. B 72, 054519 (2005).

24. Chatteriee, U. et al. Nondispersive Fermi arcs and the absence of charge ordering in the pseudogap phase of $\mathrm{Bi}_{2} \mathrm{Sr}_{2} \mathrm{CaCu}_{2} \mathrm{O}_{8+\delta}$. Phys. Rev. Lett. 96, 107006 (2006).

25. Lake, B. et al. Spins in the vortices of a high-temperature superconductor. Science 291, 1759-1762 (2001).

26. Yamada, K. et al. Doping dependence of the spatially modulated dynamical spin correlations and the superconducting-transition temperature in $\mathrm{La}_{2-x} \mathrm{Sr}_{x} \mathrm{CuO}_{4}$. Phys. Rev. B 57, 6165-6172 (1998).

27. Damascelli, A., Hussain, Z. \& Shen, Z.-X. Angle-resolved photoemission studies of the cuprate superconductors. Rev. Mod. Phys. 75, 473-541 (2003).

28. Le Tacon, M. et al. Two energy scales and two distinct quasiparticle dynamics in the superconducting state of underdoped cuprates. Nature Phys. 2, 537-543 (2006).

29. Kondo, T., Takeuchi, T., Kaminski, A., Tsuda, S. \& Shin, S. Evidence for two energy scales in the superconducting state of optimally doped $(\mathrm{Bi}, \mathrm{Pb})_{2}(\mathrm{Sr}, \mathrm{La})_{2} \mathrm{CuO}_{6+\delta}$. Phys. Rev. Lett. 98, 267004 (2007).

30. Lee, W. S. et al. Abrupt onset of a second energy gap at the superconducting transition of underdoped Bi2212. Nature 450, 81-84 (2007).

31. Smadici, S. et al. Absence of long-ranged charge order in $\mathrm{Na}_{x} \mathrm{Ca}_{2-x} \mathrm{CuO}_{2} \mathrm{Cl}_{2}(x=0.08)$. Phys. Rev. $B$ 75, 075104 (2007).

32. Abbamonte, P. et al. Spatially modulated 'Mottness' in $\mathrm{La}_{2-x} \mathrm{Ba}_{x} \mathrm{CuO}_{4}$. Nature Phys. 1 , $155-158$ (2005).

33. Meevasana, W. et al. Doping dependence of the coupling of electrons to bosonic modes in the single-layer high-temperature $\mathrm{Bi}_{2} \mathrm{Sr}_{2} \mathrm{CuO}_{6}$ superconductor. Phys. Rev. Lett. 96, 157003 (2006).

\section{Acknowledgements}

We thank J. E. Hoffman, K. M. Lang, P. A. Lee, Y. S. Lee, S. Sachdev, T. Senthil and X.-G. Wen for helpful comments. We thank C. Lindh for coding and data analysis. This research was supported in part by a Cottrell Scholarship awarded by the Research Corporation and by the MRSEC and CAREER programs of the NSF.

\section{Author contributions}

W.D.W., M.C.B. and K.C. shared equal responsibility for all aspects of this project from instrument construction to data collection and analysis. T.K. grew the samples and helped refine the STM. T.T. and H.I. contributed to sample growth. Y.W. contributed to analysis and writing of the manuscript. E.W.H. advised.

\section{Author information}

Reprints and permission information is available online at http://npg.nature.com/reprintsandpermissions. Correspondence and requests for materials should be addressed to E.W.H. 